Revista de Filosofía

ISSN: 0034-8244

https://dx.doi.org/10.5209/resf.61389

\title{
Los cuatro niveles de hermenéutica en la fenomenología de J.-L. Marion
}

Jorge Luis Roggero ${ }^{1}$

Recibido: 22 de agosto de 2018 / Aceptado: 21 de marzo de 2019

Resumen. Este artículo busca demostrar que, a partir de los lineamientos presentes en el capítulo II del reciente Reprise du donné es posible proponer cuatro niveles de hermenéutica que nos permiten resolver algunos de los problemas de la fenomenología marioniana lúcidamente planteados por Christina Gschwandtner.

Palabras clave: donación; función de la hermenéutica; usos de la hermenéutica.

\section{[en] The Four Levels of Hermeneutics in J.-L. Marion's Phenomenology}

\begin{abstract}
This article aims to demonstrate that, based on the guidelines presented in chapter II of the recent Reprise du donné, it is possible to propose four levels of hermeneutics that allow us solving some of the problems of Marionian phenomenology elegantly posed by Christina Gschwandtner.
\end{abstract}

Keywords: givenness; role of hermeneutics; uses of hermeneutics.

Sumario: 1. El enigma de la donación y de la hermenéutica. 2. La función de la hermenéutica. 3. Los cuatro niveles de hermenéutica. 4. Los niveles de hermenéutica y las críticas de Gschwandtner. 5. Referencias bibliográficas.

Cómo citar: Roggero, J. L. (2020): "Los cuatro niveles de hermenéutica en la fenomenología de J.-L. Marion”, en Revista de Filosofia 45 (1), 141-160.

$1 \quad$ Universidad de Buenos Aires jorgeluisroggero@gmail.com 
En el reciente Reprise du donné, publicado en 2016, Marion finalmente responde respecto de una crítica que había sido planteada por diversos autores a los desarrollos de la fenomenología de la donación desde la publicación de Réduction et donation (cf. Greisch 1991 y 1999, y Grondin 1992 y 1999, entre otros). La objeción puede resumirse del siguiente modo: "es indispensable aclarar si hay y cuál es la función de la hermenéutica en la fenomenología de la donación”. La formulación más reciente de esta observación y, probablemente, la que analiza en forma más detallada los diversos aspectos de la propuesta marioniana en los que deviene necesaria la introducción de una hermenéutica, se encuentra en el libro de Christina Gschwandtner, Degrees of Givenness, de 2014. Allí la autora propone la posibilidad de completar el proyecto marioniano desarrollando una gradación pormenorizada de los fenómenos y de la saturación, y prestando mayor atención a la función de la hermenéutica.

En primer lugar, Gschwandtner lamenta el reemplazo de la tópica de los fenómenos propuesta en Étant donné, en la que se presentaban diversos grados de fenomenicidad, por la nueva tópica, introducida en las últimas obras, en la que se reduce todo fenómeno a objeto o acontecimiento ( $c f$. Gschwandtner, 2014, 8-9, 39). Luego de esbozar, en Réduction et donation, la idea de una tercera reducción que consiste en una reducción a la llamada pura capaz de superar los horizontes de la objetidad (objectité) y de la enticidad (etantité) para entregarse a lo dado en sus propios términos ( $c f$. Marion, 1989, 289-305), en Étant donné, Marion ofrece una exposición sistemática de su propuesta de una fenomenología de la donación. Allí, el autor introduce una tópica del fenómeno que se corresponde con una gradación de la fenomenicidad. ${ }^{2}$ Existen tres tipos de fenómenos de acuerdo al grado en que aparecen: 1) El primer tipo es el de los "fenómenos pobres". Se trata de fenómenos que requieren sólo una intuición formal (matemáticas) o una intuición categorial (lógica). A estos fenómenos les basta para darse con la mera inteligibilidad de su concepto. Estos son los casos que, gracias a su déficit de intuición, la metafísica convierte en paradigmas de certeza epistémica abstracta (Marion, 1998, 310-311).

2) El segundo tipo es el de los “fenómenos de derecho común". Estos fenómenos se manifiestan en tanto reciben cierto cumplimiento, pero en ellos basta con una débil confirmación intuitiva del concepto para dar el fenómeno correspondiente si este se confirma repitiéndose regularmente. El caso paradigmático de fenómeno de derecho común es el "objeto técnico". En estos casos, el concepto y la intención son el "plano", "esquema" o "diseño" que permite un "mostrar por concepto' [...] que precede, determina y a veces anula la donación intuitiva” (1998, 312).

3) El tercer tipo es el de los "fenómenos saturados", fenómenos en los que la intuición desborda la expectativa de la intención. Estos fenómenos aparecen cuando se despejan las dos condiciones que se impone a la mostración de los fenómenos (el horizonte y el Yo).

2 En rigor, si bien Marion utiliza en diversos pasajes de Étant donné la expresión degrés de donation (grados de donación), corresponde hablar de "grados de fenomenicidad", pues la donación se da siempre bajo la modalidad del exceso. Según lo manifestado en otros pasajes posteriores de su obra y, por tanto, que pueden entenderse con cierto carácter correctivo, Marion enfatiza que la donación siempre se da en demasía, en exceso, con carácter saturante. En Reprise du donné se lee: "La donación se cumple siempre en demasía. La saturación de ciertos fenómenos debe entenderse como la consecuencia formal de su fenomenicidad a la medida de la donación. Una donación sin demasía se contradiría" $(2016,186)$. En este sentido, es posible afirmar que todo fenómeno, al menos potencialmente, es un fenómeno saturado. 
Esta gradación desaparece a partir de Certitudes négatives cuando la "tópica del fenómeno" es reemplazada por una nueva tabla que distingue sólo dos tipos: objetos y acontecimientos. ${ }^{3}$ Los objetos son aquellos fenómenos que son constituidos y los acontecimientos aquellos en los que se renuncia a la constitución. En su última obra, Reprise du donné, Marion repite esta nueva clasificación (Marion, 2016, 147-189). Gschwandtner lamenta esta nueva alternativa binaria que reduce la complejidad de los modos de mostración de los fenómenos. Dice la comentarista:

Esta distinción parece reinstituir los tipos de divisiones que Marion ostensiblemente intentaba superar. A pesar de que esta puede no ser una distinción absoluta, pues ambos son considerados tipos de fenomenicidad, sin embargo los 'grados' o 'variaciones' de fenomenicidad parecen haber desaparecido de la vista. Un fenómeno es o 'pobre' o 'saturado' [...] En vez de ser grados el uno del otro, estos dos parecen completamente opuestos entre sí” $(2014,39)$.

Se pasa, pues, para la autora, de una concepción gradualista de la fenomenicidad sostenida en la primera tópica del fenómeno-a una dicotómica, en la que el fenómeno sólo puede darse bajo el régimen absoluto de la saturación o bajo el régimen absoluto de la pobreza, perdiendo de vista la infinidad de matices y grados que pueden darse entre estos dos extremos.

En segundo lugar, Gschwandtner también destaca que Marion tiene una concepción equivocada de la hermenéutica, pues considera que ella debe limitarse a proveer una interpretación luego de la ocurrencia de la donación del fenómeno. La propuesta de Marion parece sostener sin más que el contexto hermenéutico y el horizonte fenomenológico constituyen limitaciones para la autodonación del fenómeno. Por el contrario, la autora subraya que los contextos y las prácticas preparatorias nos ayudan a percibir el fenómeno como saturado. "Estos contextos no pueden ser abandonados, pues constituyen una dimensión significativa de la experiencia y la manifestación misma. Sin un horizonte fenomenológico el fenómeno no puede darse a sí mismo desde sí mismo, porque no puede ser recibido. El contexto hermenéutico no restringe o ejerce control sobre el fenómeno, sino que designa la apertura y receptividad de quien se encuentra con el fenómeno" (2014, 198-199). Asimismo, Gschwandtner sostiene que el círculo hermenéutico, que se basa en tradiciones y prácticas comunitarias, es indispensable para discernir si se trata de una experiencia auténtica o inauténtica ( $c f .199)$.

En este artículo propongo como hipótesis que es posible encontrar una respuesta a las críticas de Gschwandtner a partir de las aseveraciones del propio Marion. Si bien el capítulo II de Reprise du donné, "La donation en son herméneutique", no termina de desplegar las posibilidades que su propuesta contiene, considero que

\footnotetext{
"De este modo, se dispone de una nueva tabla de fenómenos. Por un lado, los fenómenos del tipo del objeto, que comprenden los fenómenos pobres (formas lógicas, entidades matemáticas, etc.) y los fenómenos de derecho común (objetos de las ciencias de la "naturaleza", objetos industriales, etc.). Por el otro, los fenómenos del tipo del acontecimiento, que comprenden los fenómenos saturados simples (el acontecimiento en sentido estricto, según la cantidad; el ídolo o el cuadro, según la cualidad; la carne, según la relación; y el ícono o rostro del otro según la modalidad), pero también los fenómenos de revelación (que combinan diversos fenómenos saturados, como el fenómeno erótico, los fenómenos de revelación, la Revelación, etc.). Esta tabla completa y complica la de Étant donné [...], ligando saturación y acontecialidad: un fenómeno se muestra tanto más saturado cuando se da con una acontecialidad más grande" (Marion, 2010, 301, n. 1).
} 
es factible postular la existencia de cuatro niveles de hermenéutica que permiten entender la doble vertiente de la fenomenología marioniana, que retoma su talante crítico con la oposición acontecimiento / objeto, sin por eso excluir la posibilidad de grados de fenomenicidad. En esta obra, Marion señala cuatro usos diferentes de la hermenéutica, que ya se encontraban operando en la fenomenología de la donación en libros anteriores. ¿Cómo deben entenderse esta referencia a cuatro modalidades de uso? ¿Son simples ejemplos? Afirmo que no son meras alusiones defensivas de Marion, sino que en ellas puede encontrarse la indicación clara del modo en que opera su hermenéutica. Los cuatro usos constituyen una enumeración taxativa de las cuatro modalidades de hermenéutica que operan siempre, en todos los casos. Ahora bien, no todas estas modalidades tienen la misma relevancia, cabe distinguir una jerarquía entre ellas. Por este motivo entiendo que es posible hablar de cuatro niveles de hermenéutica. En un primer nivel se da lo que propongo llamar una "hermenéutica de la llamada", en el segundo una "hermenéutica de la variación", en el tercero una "hermenéutica de la gradualidad" y, finalmente, en el cuarto, una "hermenéutica infinita".

Con la finalidad de demostrar estas afirmaciones, en un primer apartado me detendré en la respuesta marioniana a la objeción hermenéutica. El fenomenólogo francés destaca que es un error considerar que existe una incompatibilidad entre la donación y la hermenéutica. Asimismo, Marion señala que la hermenéutica no impone un sentido arbitrariamente, sino que opera como una "explicitación" (Auslegung) de un sentido ya dado en la experiencia. En un segundo apartado examinaré la función de la hermenéutica en la fenomenología de la donación como una modalidad de la estructura de la llamada y la respuesta. En el tercer apartado analizaré las cuatro modalidades de uso de la hermenéutica en la obra marioniana y propondré considerarlas como niveles. Finalmente, demostraré cómo es posible contestar a las agudas objeciones de Gschwandtner desplegando ciertas posibilidades contenidas en la lógica misma del planteo marioniano.

\section{El enigma de la donación y de la hermenéutica}

En el capítulo de Reprise du donné, Marion elige responder a esta histórica objeción hermenéutica a partir de una afirmación presente en el artículo de Claudia Serban, "La méthode phénoménologique entre réduction et herméneutique", en el que la autora se pregunta si acaso la "universalidad incondicionada de la donación" no "vuelve caduco" el "recurso a la hermenéutica". Sostiene Serban: "La verdadera piedra de toque de la fenomenología propuesta por Étant donné es esta universalidad incondicionada de la donación, respecto de la cual nada se exceptúa, y que vuelve caduca, en particular, la necesidad del recurso a la hermenéutica" $(2012,88)$.

Marion señala que es un error creer que la donación se opone a la hermenéutica. En este sentido, Marion destaca que la objeción supone lo que debería probar, esto es:

la incompatibilidad de fondo de la fenomenicidad con la enunciación diferenciada de sus figuras de sentido. Esta incompatibilidad solo podría concebirse si la donación proporcionara de entrada un fenómeno objetivable, por tanto, constituido por un sentido unívoco, que no soportara ni reclamara ninguna interpretación $(2016,62)$. 
La donación marioniana no puede ser equiparada a una causalidad eficiente que produce un objeto. Marion destaca que el dar de la donación no consiste en una producción o una eficiencia. La donación ostenta un carácter enigmático: "surge menos como una respuesta que como una pregunta, menos como un argumento final que como una indecisión, en espera" $(2016,63)$. Se puede saber lo que no es: sabemos que no actúa como una causa eficiente, y que no se limita a la intuición sensible, porque no se confunde con la intuición, sino que constituye una instancia anterior. Sin embargo, no es posible decir lo que es, pues la única determinación aceptable respecto de la donación es afirmar su indeterminación (cf. 2016, 78). Frente a este carácter de la donación, que no admite ningún sentido unívoco, sino que "abre y da" (Henry, 1991, 13), Marion se pregunta:

¿No será posible que la hermenéutica, lejos de desaparecer con la donación (o de hacerla desaparecer al comenzar a hablar), solo se despierte al responder a la palabra que la cumple? $(2016,68)$

Es la indeterminación misma de la donación la que reclama la intervención de la hermenéutica.

En los $\S \S 11$ a 13, Marion se detiene en un análisis de la "crítica al mito de lo dado" con el objetivo de señalar que el hecho de que lo dado esté mediado no implica que deba constituirse como objeto.

Desde el punto de vista de una fenomenología rigurosa, conviene sostener la paradoja de que pertenece a lo dado no darse inmediatamente $\mathrm{y}$, sobre todo, no darse en la inmediatez de los sense data; aunque se dé en una perfecta facticidad, o más bien porque se da como un factum incondicionado y originario $(2016,74)$.

Apoyándose en el ejemplo heideggeriano de la vivencia de la cátedra, Marion subraya la particularidad de la propuesta fenomenológica contra las presuposiciones del constructivismo y el empirismo:

Lo que se encuentra dado no son los sense data, impropios inmediatos, inmediatos abstractos y derivados: lo dado inmediato no consiste jamás en el color de la madera, ni en las dimensiones reales del mueble, ni en los detalles de su forma, ni los efectos que en el produce la luz de la mañana, ni la resonancia de los sonidos de la voz, etc. Nada de todo eso aparece de entrada o, más exactamente, todo eso aparece (o mejor, aparecerá) luego, gracias a la mediación del primer apareciente, el que me aparece como la cátedra profesoral misma. [...] Lo inmediato consiste en una significación, que mediatiza todas las vivencias $(2016,77)$.

Marion concluye el $\S 13$ señalando que hay que considerar el "problema de la donación" como un enigma que se sitúa más allá de la dicotomía planteada por la "crítica del mito de lo dado". La donación no puede ser reducida ni a lo inmediato en el sentido de los sense data de la impresión subjetiva, ni a lo mediato de la objetidad construida por la consciencia (cf. 2016, 77-78).

Una vez más hay que recordar escuchar la pregunta: „Was heißt ,gegeben', ,Gegebenheit' - dieses Zauberwort der Phänomenologie und der ,Stein der Anstoßes' bei den Anderen?“, 
y, por tanto, permanecer en el enigma. La indeterminación de lo dado ofrece quizás su única determinación correcta, la que lo distingue de todo lo que lo sigue: los sense data, los objetos, los conocimientos, [todos ellos] brotes de su acontecimientos $(2016,78)$.

Lo que se da, se da como significación, nos rodea un aparecer que se da "bajo la figura y los escorzos de una significación" (Marion, 2016, 79). Marion da algunos ejemplos. No se percibe un sonido puro, sino el murmullo de un torrente de montaña de un río en particular o el ruido de un motor de un autor en particular. No se percibe el color amarillo, sino una parte de un muro de ese color. No se percibe el color azul, sino un azul particular (el de Cézanne). No se percibe el gusto del vino, sino el gusto de un vino de Borgoña, de tantos años, etc.

En todos los casos, solo percibo si una significación abre el campo al aparecer de sensaciones puras; y es por este motivo que la cosa solo aparece por escorzos, porque la significación, cumplida y visible de entrada para el espíritu, la mayoría de las veces (al menos en el caso de fenómenos de derecho común) debe esperar que los escorzos, siempre parciales y a completar, tomen lugar y poco a poco la validen. Tal inversión del orden de la donación impone, por tanto, que lo dado, en el sentido de sense data, no tenga ninguna validez fenoménica inmediata, no abra aún a la fenomenicidad como tal, porque depende de una significación, ya sea anterior (como en el ejemplo de Heidegger) o sea aún esperada (como en el caso de la descripción de Husserl) $(2016,79)$.

Marion sugiere que, en este sentido, la hermenéutica no desaparece ante la donación, sino que, por el contrario, debe intervenir "respondiendo al enigma de los sense data por el recubrimiento de su significación" $(2016,79)$. Pero esta hermenéutica no debe ser pensada como aquella que trae la solución, que puede ofrecer la determinación desde una decisión arbitraria ante la indeterminación de lo dado y la donación. Ciertamente, es la indeterminación de la donación la que demanda una hermenéutica, pero esta hermenéutica también posee un carácter enigmático, pues provee una "interpretación" (Auselgung) que -según Heideggerdepende de la "comprensión" (Verstehen) como existenciario (cf. Marion, 2016, 7980). Explica Marion:

La primer prudencia consiste en no recurrir a la hermenéutica como la solución universal para determinar el sentido de lo dado, como si ésta fuera de suyo y cayera del cielo inteligible sobre algo dado oscuro y problemático; pues el acto de interpretación no va más de suyo que la recepción de lo dado, con la que comparte el "carácter enigmático". Pues, ya que, en este caso, la hermenéutica no opera sobre objetos, ni sobre sense data, por tanto, ella no consiste jamás en una interpretación pura y simple, libre [...] y que, por autoridad arbitraria, modificaría a voluntad el sentido del aparecer de la cosa misma $(2016,80)$.

Marion opone su noción de hermenéutica a la ideología. ${ }^{4}$ Mientras que la actitud de la ideología se caracteriza por imponer un sentido con el único criterio de que éste le sea útil a sus fines, la hermenéutica actúa de modo inverso, pues procura

4 Marion introduce esta cuestión sin ahondar demasiado en ella, sin referir al debate entre Gadamer y Habermas ( $c f$. Habermas, 1971), ni a las reflexiones ricœurianas en torno a la ideología (cf. Ricœur, 1986, 333-392). 
reencontrar $e l$ sentido que demanda por sí mismo aquello que demanda interpretación. El sentido que la hermenéutica (re)encuentra para lo que interpreta no viene del ego, sino de la cosa misma a la espera de interpretación. [...] No podríamos imaginar una actividad del ego hermeneuta hacia una materia pasiva a interpretar. En cierto sentido, el ego debe permanecer pasivo para recibir $\mathrm{el}$ sentido que conviene exactamente a lo que demanda interpretación; el sentido viene activamente a lo interpretable y a partir de sí mismo, el intérprete debe solo dejar desplegarse lo que se encuentra implícitamente disponible en y sugerido por lo dado mismo. [...] El hermeneuta no da un sentido a lo dado, fijándolo y decidiéndolo, sino que, cada vez, le deja desplegar su propio sentido, es decir, el que lo hace aparecer como él mismo, como un fenómeno que se muestra en sí y para sí. El sí del fenómeno regula en última instancia toda la donación de sentido: no se trata de constituir algo dado en un objeto para el ego, sino de dejar venir al fenómeno su propio sentido, descubierto más que inventado, reconocido más que conocido, por la intermediación circunstancial del ego. El sentido que hace visible la hermenéutica no proviene tanto de la decisión del hermeneuta, como del fenómeno mismo, respecto del cual el hermeneuta es el descubridor y, por tanto, el servidor, jamás el autor ni el propietario $(2016,81)$.

Marion entiende la hermenéutica como la operación metodológica por medio de la cual el adonado se entrega al fenómeno y deja que éste determine su propio sentido. En una entrevista que tuvo lugar en su domicilio personal en Paris, el 16 de noviembre de 2016, Marion me dijo que, de algún modo, él entiende que la hermenéutica es la operación opuesta a la constitución. Puede afirmarse, siguiendo su sugerencia, que su hermenéutica pone en práctica una suerte de Sinngebung invertida, una Sinngebung que se limita a una tarea de "explicitación" (Auslegung) del sentido ya dado. ${ }^{5}$

\section{La función de la hermenéutica}

Con este Sinngebung invertida, se da un desplazamiento de la autoridad interpretativa que pasa del intérprete al interpretado. Este movimiento se observa claramente, según Marion, en la idea gadameriana de "fusión de horizontes". Nietzsche plantea la aparentemente insuperable aporía de la ciencia histórica, que decide la imposibilidad

\footnotetext{
Sin embargo, cabe destacar que el proceso de dación de sentido en Husserl reviste una complejidad particular, que si bien culmina en una constitución activa por parte del yo, también registra diversas etapas pasivas. A partir de un análisis genético, Husserl establece que en los primeros estratos, puede advertirse que el dato hylético se organiza por sí mismo. El sentido se organiza desde la hýle, desde el polo objetivo. En los Manuscritos C, Husserl habla de una "llamada" (Anruf) de la sensación que despierta la atención y la acción del yo ( $c f$. HuaM VIII, 191ss, 350-351). De algún modo, esta "llamada" da el puntapié inicial para el complejo proceso de formación del sentido perceptual, en el que intervienen, en una primera instancia, síntesis temporales, kinestésicas y asociativas ( $c f$. Welton, 1983, 232-253). Explica Roberto Walton: "El complejo hylético no solo está sujeto a una síntesis temporal y asociativa, sino que depende de una síntesis kinestésica porque es reconfigurado por los movimientos del cuerpo propio. La organización temporal y asociativa indicada por Husserl es complementada por una configuración kinestésica que produce una fusión de contenidos en virtud de la cual se vuelve innecesaria una aprehensión noética especial. La captación no tiene los caracteres asignados a la aprehensión noética en la fenomenología estática porque se enfrenta con un dato material que ejerce una atracción y está ya organizado. Por eso tiene más bien el carácter de una constatación antes que el de una interpretación. [Y compartiendo la opinión de Don Welton, a continuación, Roberto Walton lo cita] Welton concluye que en tales síntesis se encuentra 'el origen de los sentidos perceptivos y del horizonte perceptivo en la fenomenología de Husserl"” (Walton, 2015, 211-212).
} 
misma para la historia de realizar su tarea, pues si interpreta a partir del horizonte del intérprete destruye el horizonte de lo interpretado y si interpreta a partir del horizonte de lo interpretado destruye el horizonte del intérprete ( $c f$. KSA I, 258-270). Frente a este conflicto de horizontes, Gadamer propone una solución: la hermenéutica solo deviene factible si los dos horizontes se encuentran y se fusionan.

El horizonte del presente no se forma entonces en absoluto sin el pasado. No hay más horizonte del presente, que pueda existir por separado, que los horizontes históricos que deberíamos conquistar. Por el contrario, la comprensión [Verstehen] es siempre el proceso de fusión [Vorgang der Verschmelzung] de tales horizontes supuestamente independientes el uno del otro (Gadamer, 1999, 311)

Pero ¿cómo opera esta "fusión de horizontes"? Gadamer aclara que la comprensión de una tradición opera por medio del "desplazarse" (sichversetzen), pero este desplazarse no anula ni el horizonte propio ni el horizonte pasado: es poniéndose en el lugar del otro, sin dejar de ser uno mismo, que es posible advertir la irreductibilidad de esa alteridad:

Para comprender una tradición, sin duda, hay que tener un horizonte histórico. Pero no puede tratarse de adquirir este horizonte transportándose a una situación histórica. Por el contrario, siempre hay que tener ya un horizonte para poder así desplazarse en una situación. Pues, ¿qué significa "desplazarse"? Sin ninguna duda, esto no significa simplemente hacer abstracción de sí. Sin duda hay que hacerlo bien, en la medida en que debe realmente representarse la otra situación. Pero, en esta otra situación, justamente, también es necesario introducirse uno mismo. Solamente entonces el acto de desplazarse toma su sentido pleno. Desplazándose, por ejemplo, al lugar del otro, se lo comprende, es decir, es precisamente poniéndose uno mismo en el lugar del otro que tomamos consciencia de su alteridad, más bien, de su irreductible individualidad (Gadamer, 1999, $310)$.

Pero esta fusión, destaca Marion, supone un actuar recíproco entre lo dado (el horizonte pasado) y el fenómeno (el horizonte presente). ¿Cómo puede darse esta reciprocidad? Marion señala que ésta puede advertirse en la idea gadameriana de "diálogo". Explica Gadamer:

Volvemos, pues, a lo que hemos constatado: el fenómeno hermenéutico lleva en sí igualmente la originariedad del diálogo y de la estructura pregunta-respuesta. El que un texto transmitido se convierta en objeto de la interpretación quiere decir para empezar que plantea una pregunta al intérprete. La interpretación contiene en esta medida una referencia esencial constante a la pregunta que se le ha planteado. Comprender un texto quiere decir comprender esta pregunta. Pero esto ocurre, como ya hemos mostrado, cuando se gana el horizonte hermenéutico (Gadamer, 1999, 375).

Marion señala que, en la interpretación histórica gadameriana-que finalmente es una interpretación de texto--, se da la lógica recíproca del diálogo, que se refleja en la estructura de la pregunta y la respuesta. Esto conlleva -según Gadamer- una mutua imbricación: 
Pues la dialéctica pregunta-respuesta, que hemos puesto a la luz, hace aparecer a la comprensión como una relación recíproca semejante a la de un diálogo. Ciertamente, un texto no nos habla como un tú. Primero debemos nosotros, los que comprendemos, llevarlo al discurso desde nosotros. Ahora bien, como se ha mostrado, tal modo comprensivo de llevar-al-discurso [verstehendes zum-Reden-bringen] no consiste en una intervención arbitraria de iniciativa personal: ella se refiere a su vez como pregunta a la respuesta esperada del texto (Gadamer, 1999, 383).

La pregunta respecto del sentido de lo dado, por la que -como hemos analizadolo dado se fenomenaliza, solo alcanza su sentido en la respuesta "que no viene, en última instancia, del intérprete, sino de lo interpretado, del texto" (Marion, 2016, 83). Pero, entonces, la hermenéutica constituye un caso "particular y ejemplar" del juego entre la llamada de lo dado y la respuesta por medio del sentido de lo que se muestra. Marion postula entonces una primera tesis:

La hermenéutica debe entenderse en acuerdo con lo dado, bajo las figuras de la llamada y la respuesta. Lejos de que la hermenéutica sobrepase la donación o la sustituya, ella se despliega ahí, como un caso de la relación originaria entre lo que se da y lo que se muestra $(2016,84)$.

De este modo, ya en esta primera tesis, Marion señala que la estructura de la llamada y la respuesta -propia de la tercera reducción y del modo de donación de todos los fenómenos $-{ }^{6}$ es una estructura hermenéutica, pues responde a la estructura de la pregunta y la respuesta. La donación ostenta una dimensión hermenéutica originaria en tanto se da bajo el modo de la llamada y se muestra en la respuesta.

Atento a esta consecuencia de su primera tesis, Marion continúa su reflexión explicando la relación entre fenomenología y hermenéutica:

Si la hermenéutica encuentra su espacio y su legitimidad en la función de responder a la pregunta que plantea lo dado, fijándole $s u$ sentido propio, entonces no hay que hablar de un estatuto hermenéutico de la fenomenología (como si ésta última se inscribiera en aquella

6 En Réduction et donation, Marion sostiene que la reducción heideggeriana, cuya epoché se manifiesta en el temple anímico de la angustia, opera a partir de una reinvidicación (Anspruch), de la llamada del ser. Siguiendo a Lévinas, Marion entiende que puede encontrarse una instancia más originaria que el ser desde la cual se dé la reivindicación. Pero, entonces, si esto es así, es posible ir más allá del planteo levinasiano también y afirmar que más originaria que la llamada del otro, es la estructura misma de la llamada (Marion, 1989, 295-296). Marion propone: "Ahora bien, la reconducción de la reivindicación del ser a la forma pura de la llamada que, por otra parte, es la única que la hace posible, repite también la reducción: más esencial que la reducción de los objetos a la conciencia de un Yo, intervenía su reducción al rango de entes, y por lo tanto la reducción de los entes al Dasein como único ente ontológico; más esencial aún se afirmaba la reducción de todos los entes al ser, al reivindicar la puesta en juego del Dasein; más esencial, en fin, que esta (reducción por) reivindicación aparece finalmente la reducción de toda reivindicación a la forma pura de la llamada. Luego de la reducción trascendental y la reducción existencial, interviene la reducción a y de la llamada. Lo que se da solo se da a aquel que se entrega a la llamada bajo la forma pura de una confirmación de la llamada reproducida en tanto que recibida" (1989, 296). Y más adelante: "La llamada aparece de este modo como el esquema originario de las dos reducciones anteriores, precisamente porque solo ella permite reconducir a..., en lo que se exige entregarse a lo dado de la llamada como tal: rendirse a la llamada, en el doble sentido de abandonarse a ella y de desplazarse hacia ella. En tanto que pura reducción -dado que es perfecta reconducción a...-, la llamada que reivindica para sí misma incumbe eminentemente a la fenomenología” (296-297). La tercera reducción, como reducción a la llamada pura, finalmente revela la estructura que constituye el núcleo de toda reducción. 
como una de sus derivaciones), sino que hay que reconocer el estatuto radicalmente fenomenológico de la hermenéutica: la hermenéutica constituye una de la operaciones esenciales de la fenomenología, al igual que la intencionalidad, las reducciones y la constitución, y solo así mantiene su plena autoridad (2016, 84).

La fenomenología de la donación tiene una dimensión hermenéutica fundamental, pero esta hermenéutica se subordina a los lineamientos de la fenomenología. Para explicar esta subordinación, Marion se detiene en el análisis heideggeriano de la relación entre la "comprensión" (Verstehen), la "explicitación” (Auslegung) y el "enunciado" (Aussage) en Sein und Zeit.

En el $\S 32$, Heidegger establece la dependencia de la "explicitación" respecto de la "comprensión": "La explicitación se funda existencialmente en la comprensión, y no es ésta la que llega a ser por medio de aquélla" (GA 2, 197). La explicitación se funda en la comprensión en tanto existenciario del Dasein. La "circunspección" (Umsicht) explicita el mundo ya comprendido, ya abierto por la comprensión. El trato con lo "a la mano" (das Zuhandene) se da en la "circunspección" comprensora que explicita su "para qué" (Um-zu). Lo explicitado, es decir, lo comprendido expresamente tiene la estructura del "algo en tanto que algo" (Etwas als Etwas). El "en tanto que" (als) es el modo en que se explicita lo comprendido ( $c f$. GA 2, 198).

En el $\S 33$, Heidegger se detiene en el "enunciado" como una forma derivada en la que se cumple la "explicitación". Heidegger destaca que el enunciado tiene un "sentido" (Sinn), pero éste no radica en el acto de juzgar. Es posible entender el enunciado de tres modos distintos:

1) El enunciado puede ser entendido en su sentido propiamente apofántico, como "señalamiento" (Aufzeigung) de un ente. Cuando decimos "el martillo es demasiado pesado", lo que se descubre a la visión no es un "sentido", sino un ente bajo la modalidad de lo "a la mano".

2) Asimismo, el enunciado puede ser entendido como "predicación" (Prädikation). Siguiendo el mismo ejemplo, en el enunciado "el martillo es demasiado pesado", lo enunciado sigue siendo el martillo, pero está reducido, determinado por el predicado determinante "demasiado pesado". La predicación hace que nuestra mirada se oriente sobre cierto aspecto del ente.

3) Finalmente, el enunciado también puede ser entendido como "comunicación" (Mitteilung). El enunciado permite transmitir lo señalado y lo enunciado a otro.

De este modo, teniendo en cuenta las tres acepciones, un enunciado es un "señalamiento que determina y comunica" (GA 2, 208). Sin embargo, el enunciado no puede ser comprendido a partir de estas definiciones y de la especificación de sus funciones. Heidegger entiende que es necesario explicitar qué es lo que el enunciado presupone. Es decir, corresponde entender al enunciado en su dependencia respecto de la explicitación y de la comprensión. Comentando estos parágrafos de Sein und Zeit, Marion destaca:

La hermenéutica que ignora su estatuto originario de interpretación del Dasein por sí mismo se rebaja a la trivialidad en una simple, arbitraria e ilegítima enunciación de alguna cosa a propósito de alguna cosa $(2016,85)$.

El plano lógico, el plano de la enunciación está precedido por y se funda sobre el ontológico. Heidegger acepta la tesis husserliana respecto del carácter derivado de 
las significaciones de orden lingüístico. El enunciado no puede ser nunca arbitrario, pues debe presuponer el ser-en-el-mundo, el estar ya en contacto con las cosas sobre las que va a pronunciarse. En palabras de Heidegger: "El enunciado no es un comportamiento en el aire que por sí mismo pudiera abrir primariamente el ente, sino que se mueve ya siempre sobre la base del ser-en-el-mundo" (GA 2, 208). Es precisamente porque el Dasein ya está implicado en el mundo y el existenciario de la comprensión ya está operando, que es posible un enunciado. Los enunciados proposicionales son formas derivadas respecto de nuestra experiencia del mundo y su comprensión, que es más originaria. Es más, dice Heidegger:

El trato circunspectivo-explicitante con el ente a la mano del mundo circundante, que lo "ve" en cuanto mesa, puerta, coche o puente, no tiene necesidad de exponer también en un enunciado determinativo lo circunspectivamente explicitado. Toda simple visión antepredicativa de lo a la mano ya es en sí misma comprensora-explicitante (GA 2, 198).

La explicitación puede darse sin necesidad de recurrir a un enunciado. Por ejemplo, comprendemos de modo antepredicativo la "pesadez" del martillo sin necesidad de recurrir a ningún enunciado. Su pesadez queda explicitada cuando lo dejamos y tomamos otro más liviano y adecuado para el trabajo que queremos realizar (cf. GA 2, 208-209). Explica Heidegger:

El modo originario de cumplimiento de la explicitación no consiste en la proposición enunciativa teorética, sino en el hecho de que en la circunspección del ocuparse se deja de lado o se cambia la herramienta inapropiada "sin decir una sola palabra". De la falta de palabras no se debe concluir la falta de explicitación (GA 2, 209).

Heidegger postula, pues, el enraizamiento profundo de la hermenéutica en la fenomenología. La base para la interpretación bajo la modalidad enunciativa está dada por esta comprensión antepredicativa que determina su validez, porque en ella está su sentido. El "en tanto que" apofántico debe basarse en el "en tanto que" hermenéutico-existencial.

El enunciado y la estructura del enunciado, vale decir, el "en tanto que" apofántico, están fundados en la explicitación y en su estructura, esto es, en el "en tanto que" hermenéutico, y, más originariamente aún, en la comprensión y en el estado de abierto del Dasein" (GA 2, 295).

Marion sostiene que su hermenéutica debe entenderse en este sentido heideggeriano, como enraizada en la fenomenología. Ahora bien, cabe entonces hacer una aclaración. Si el enunciado y la explicitación reenvían a la comprensión como existenciario del Dasein, entonces el sentido no proviene del fenómeno, sino del Dasein. Marion prevé esta objeción y propone que la respuesta está en el modo en el que se comprenda el "circulo hermenéutico":

...hay un círculo, pero no tiene nada de vicioso y, más que intentar evitarlo hay que ser cuidadoso para entrar del modo correcto. Se trata, por otra parte, menos de un círculo que de una relación, más exactamente, se trata de lo que Gadamer llama una palabra recíproca (Gespräch), el juego de la pregunta con la respuesta $(2016,87)$. 
Marion explica que si bien la hermenéutica de la cosa se origina en la comprensión de sí del Dasein, el Dasein es un ser-en-el-mundo, y su posibilidad se juega en ese estar y exponerse en el mundo. De este modo, se establece una reciprocidad entre el sentido del Dasein y la significación de cada ente, "la comprensión (Verstehen), en tanto permite la interpretación (Auslegung), se juega en la 'estructura de la pregunta y de la respuesta' (Gadamer)" (Marion, 2016, 87). Y esta estructura es la estructura de la llamada y la respuesta. Dice Marion:

Si, efectivamente, la recepción y la identificación de lo dado implican que esto dado permanezca siempre pendiente de interpretación en tanto fenómeno dotado de significación, entonces la instancia hermenéutica fija el lugar de lo dado porque ella se fija ahí a sí misma. Hay que comprender a la hermenéutica misma como encargada de esta recepción y de esta identificación de lo dado (2016, 87-88).

Marion explicita de modo claro el lugar de la hermenéutica en su fenomenología. Ella se ubica en la donación misma como encargada de la recepción y la identificación de lo dado. Y esta tarea es fundamental porque no todo lo que se da se muestra. Existe una "distancia" (écart) entre lo que se da y lo que se muestra que responde al carácter infinito de la donación y al carácter finito del adonado receptor. Por este motivo, la hermenéutica cumple una función fundamental en la fenomenología de la donación.

La hermenéutica gestiona la distancia entre lo que se da y lo que se muestra, interpretando la llamada (o, frecuentemente, intuición) por la respuesta (concepto o significación) (Marion, 2016, 89).

La hermenéutica tiene la tarea decisiva de administrar el pasaje de la donación a la mostración.

Marion concluye retomando la objeción de Serban, no sólo la donación no vuelve caduca a la hermenéutica, sino que la fenomenología de la donación solo puede permitir que los fenómenos se muestren como dados en la medida en la que ponga en práctica una hermenéutica que dé cuenta de lo dado "en tanto que" mostrado ( $c f$. 2016, 89).

Para finalizar, Marion destaca que "la hermenéutica no se agrega a la fenomenología", sino que constituye una dimensión propia de la fenomenología porque "sin significación, lo dado aplazaría su mostración" $(2016,90)$.

\section{Los cuatro niveles de hermenéutica}

En el $\S 17$, parágrafo final del capítulo II de Reprise du donné, Marion señala que esta noción de hermenéutica que presentó en los parágrafos precedentes puede encontrarse ya operando en su obra fenomenológica desde sus comienzos. Marion destaca cuatro apariciones. Cada una de ellas refleja una modalidad distinta de uso, pero no todas parecen tener la misma importancia. Por este motivo, propongo hablar de niveles de hermenéutica.

1) El primer uso es el fundamental y, por lo tanto, puede ser identificado como el primer nivel: el de la "hermenéutica de la llamada". En este caso, la hermenéutica opera directamente sobre la donación, decidiendo si ésta tuvo o no lugar al decidir si la llamada es efectivamente una llamada para el adonado o no. Dice Marion: 
Por tanto, escucho la llamada en y por mi interpretación: debí determinar que ahí había una llamada, y determinarme como su destinatario; entonces solamente será permitida la respuesta, aunque sea una denegación. Y solo mi interpretación de la llamada permitirá esta respuesta, confirmando así la regla que dicta que la llamada solo se hace escuchar en la respuesta, que decide no solamente del contenido de la llamada, sino en primer lugar de su efectividad (o de su carácter ilusorio) $(2016,91)$.

En este sentido, la posición fenomenológica asumida por Marion implica complejizar la relación entre el hecho y la interpretación. Jocelyn Benoist acusa a Marion de sumarse a un planteo "postmoderno" por el que se olvidaría la independencia, la distancia y la exterioridad de la llamada respecto de la respuesta, es decir, se olvidaría la diferencia entre el hecho y la interpretación ( $c f$. Benoist, 2016, 191-198). Cabe señalar que para Marion, y para la fenomenología en general, no se trata de afirmar nietzscheanamente que "no hay hechos, sino interpretaciones"; no se trata de introducir un sentido mediante la interpretación frente a la ausencia de sentido de los hechos, frente al sinsentido de la realidad. La fenomenología (Husserl y Heidegger) advierte una dimensión de sentido que ya está presente en la experiencia de las cosas mismas. Como hemos analizado en el apartado anterior respecto de Heidegger, el enunciado -cuando no es una expresión arbitraria- no hace más que recoger el sentido ya dado en la experiencia antepredicativa. La crítica de Benoist parece ser planteada a partir de la aceptación de la crítica analítica a la fenomenología respecto de su concepción del sentido. La filosofía analítica critica el uso de las nociones de "sentido" y "significación” más allá de la esfera lingüística, porque acepta como un hecho la idea tradicional de una separación tajante entre la esfera del lenguaje y los significados, y la esfera de los hechos brutos desprovistos de significación. Como distingue con gracia Claude Romano:

Tome el caso de un filósofo, examine el empleo que hace del término "sentido" e inmediatamente usted no tendrá dudas: si solo habla de dar un sentido a un signo y de explicar el sentido de una frase, usted tiene un filósofo analítico; si emplea "sentido" de una manera más amplia usted está tratando con un discípulo de Husserl (2010, 134-135).

La hermenéutica marioniana tiene como primera tarea determinar por la respuesta, el contenido y la efectividad misma de la llamada, pero esta labor es solo realizable si se advierte la responsabilidad por la fenomenicidad desde una implicación con las cosas que anula toda distancia y exterioridad; y en esta implicación, en ese estar ya en contacto con las cosas, se da la decisión hermenéutica sobre la existencia de la llamada. Ahora bien, teniendo en cuenta la universalidad de la donación (Marion sostiene la donación de la ausencia misma), la respuesta que decide, decide principalmente sobre su responsabilidad personal ante lo dado concreto. Esta decisión nunca es arbitraria, pues se basa en un estar ya siempre en contacto con las cosas y obtiene su sentido de esa experiencia.

Si bien Marion no lo señala, este primer nivel de hermenéutica se relaciona con la decisión inmanente y con la "hermenéutica imperial" a la que se hace referencia en el § 29 de Étant donné (cf. 1998, 419-423). El adonado, ante la llamada, se enfrenta a una decisión fundacional, capaz de inaugurar una nueva lógica, pues tener el coraje de decidir recibir lo dado en tanto tal no puede basarse en un cálculo según la racionalidad del intercambio económico. Probablemente por este motivo, Marion 
relaciona la hermenéutica de la llamada con la fenomenología del don. El don -en su gratuidad-aparece en la respuesta que decide sobre su existencia, pero esta respuesta se conforma de diversas interpretaciones en las que se asume ciertamente un riesgo.

A diferencia del intercambio y del comercio - que me dispensan de toda interpretación y que de entrada significan que hay un objeto ya disponible para intercambiar, vender o comprar (está expuesto en un negocio), a un precio (fijo o a negociar, poco importa), con una utilidad y un valor de uso patente (aunque sea eventualmente ilusorio)-, ningún ente u objeto ofrece en sí mismo un don (Marion, 2016, 91).

Para entender que un fenómeno se da como un don, es necesario poner en juego una hermenéutica que lo interprete como don y que interprete para quién está dirigido.

2) El segundo uso de la hermenéutica se da ante el fenómeno saturado específicamente, pues, para ser aprehendido, éste demanda una pluralidad de conceptos o significaciones. En este sentido, considero que se trata del cuarto nivel de hermenéutica, pues su aplicación opera luego de que actúen los otros tres niveles. En este caso, la hermenéutica cumple una doble función: en primer lugar, persigue las significaciones faltantes, pero, en segundo lugar y principalmente, interviene para admitir que hay significaciones faltantes, pues no estamos ante un fenómeno cognoscible bajo la modalidad de la objetidad (cf. Marion, 2016, 92). En este nivel se constata, pues, que ante un fenómeno saturado siempre es posible otra interpretación.

Este uso, recuerda Marion, puede encontrarse en De surcroît, cuando se invoca una "hermenéutica infinita" ante las infinitas interpretaciones posibles que demanda la intuición saturante del fenómeno del rostro.

El rostro del otro requiere una hermenéutica infinita, equivalente al "progreso al infinito" de la moralidad según Kant. Pues todo rostro exige la inmortalidad (Marion, 2010a, 159). ${ }^{7}$

En estos casos, sostiene Marion, la hermenéutica retoma la función que la constitución ya no puede asumir (pues ella se restringe a los límites de la objetidad).

3) El tercer uso de la hermenéutica, que se corresponde con el tercer nivel, con el nivel de la "hermenéutica de la gradualidad", tiene que ver con la tarea de distinguir los grados de intuición que permiten saber si estamos ante un fenómeno pobre, ante un fenómeno de derecho común o ante un fenómeno saturado. Pero ¿cómo es posible esta distinción? ¿Se trata de categorías fijas?

Marion contesta que este tipo de preguntas responden a una matriz metafísica que presupone que los grados constituyen compartimentos estancos, que entre ellos se dan fronteras infranqueables (regiones) que no admiten pasajes ni transiciones. Por el contrario, argumenta Marion, la fenomenología de la donación admite una gradualidad de la saturación "ya que lo dado intuitivo puede terminar por mostrarse (fenomenalizarse) como más o menos saturado, según la hermenéutica que la considere" $(2016,94)$.

El ejemplo para advertir esta gradualidad, y el carácter flexible de lo grados, es el mismo que fue utilizado en « La banalité de la saturation »: el cuadro 212 de Rothko ( $c f$. Marion, 2005b, 158-159). Podemos ver la obra como tres bandas

Marion define al otro, en la entrevista para la revista Le Philosophoire, en los siguientes términos: "Otro, o eso con lo que jamás se ha terminado" (2005a, 17). 
de colores diferentes dispuestas en sentido horizontal y darle la significación de un objeto (una bandera), o podemos advertir que se trata de un fenómeno saturado con las características del ídolo. El pasaje entre la objetidad y la saturación permanece abierto y transitable; la saturación es banal, pues puede encontrarse en los fenómenos más pobres ( $c f$. Marion, 2016, 94).

La tematización de este uso por parte de Marion es un poco insuficiente, pues no parece distinguirse con claridad del cuarto uso en el que se estable la distinción entre acontecimiento y objeto. Sin embargo, en este tercer nivel, lo decisivo es el acento puesto en la posibilidad de gradación de la fenominicidad, mientras que en el cuarto uso (nivel de la "hermenéutica de la variación"), como analizaremos a continuación, el énfasis se sitúa en establecer críticamente el régimen de fenomenicidad que corresponde a cada momento (objetidad o acontecialidad).

4) El cuarto uso, que propongo situar en el segundo nivel de importancia, en el nivel de la "hermenéutica de la variación", es el que opera a partir de la nueva tópica de los fenómenos que distingue sólo entre dos modos de fenomenicidad: objetos y acontecimientos. La hermenéutica, en este nivel, actúa poniendo en práctica las "variaciones hermenéuticas" presentadas en Certitudes négatives, que permite la transformación de un objeto en acontecimiento y viceversa. ${ }^{8}$ Se trata de un uso crucial, pues una vez aceptada la responsabilidad por la llamada, cabe distinguir si se trata de un objeto o un acontecimiento, y permanecer vigilante respecto de la necesidad de introducir una "variación hermenéutica".

\section{Los niveles de hermenéutica y las críticas de Gschwandtner}

Estos niveles de hermenéutica nos permiten extraer algunas conclusiones y encontrar algunos lineamientos para responder a las lúcidas objeciones de Christina Gschwandtner. ${ }^{9}$ En primer lugar, la hermenéutica marioniana en su diversos niveles prevé distintos tipos de uso que combinados permiten advertir cómo es posible articular una gradación de la fenomenicidad con una opción dicotómica entre objetos y acontecimientos.

En el primer nivel, la decisión fundamental, la "decisión inmanente" está dada por la "hermenéutica de la llamada" que pone en acción a la "hermenéutica imperial", que decide por medio de la respuesta que allí hubo una llamada. Esta respuesta, que gestiona el pasaje de la donación a la fenomenalización, inaugura una nueva racionalidad, pues implica la aceptación de la incomensurabilidad de la donación.

En el cuarto nivel, presentado por Marion como un segundo uso, opera una hermenéutica de la saturación que tiene la tarea de -ante el fenómeno saturado-

\footnotetext{
"La distinción de los modos de la fenomenicidad (para nosotros entre objeto y acontecimiento) puede articularse sobre variaciones hermenéuticas" (Marion, 2010, 307).

9 Cabe destacar que la publicación de Reprise du donné (2016) es posterior a la publicación del libro de Gschwandtner, Degrees of Givenness (2014). En el libro se hace una breve mención a la conferencia para las Père Marquette Lectures, "Givenness and Hermeneutics" (2013), que constituye la segunda versión del capítulo II de Reprise du donné, "La donation en son herméneutique » (la primera versión es la de la conferencia «Quelques précisions sur la réduction, le donné, l'en herméneutique et la donation », pronunciada con ocasión del coloquio sobre la obra de Gondek y Tengelyi, Neue Phänomenologie in Frankreich, el 8 de marzo de 2012, y publicada en 2014). Gschwandtner considera que la conferencia, que ya esboza sucintamente los cuatro usos, se limita a "reafirmar el rol interpretativo de la hermenéutica y la prioridad de lo dado" (Gschwandtner, 2014, 24).
} 
advertir la pluralidad de significados que éste demanda y entregarse pues a la labor de una "hermenéutica infinita". Esta concepción de la hermenéutica es la que da lugar a la crítica de Gschwandtner. La necesidad de recorrer la distancia entre la intuición saturante y la insuficiencia de cada significado posible, que implica, ante todo, reconocer que estamos frente a un fenómeno irreductible a la objetidad, es decir, irreductible a un significado único y fijo, también parece sugerir que todos los significados están en pie de igualdad. Según Gschwandtner, la "certeza negativa"10 y la hermenéutica están conectadas en cierto nivel. Son necesarias infinitas interpretaciones porque la razón nunca será capaz de aprehender al fenómeno saturado (cf. 2014, 18). Pero de este modo, pareciera que no es posible establecer una jerarquía entre las interpretaciones.

Podemos proveer muchas interpretaciones, porque el acontecimiento es incomprensible. Pero, esto sugiere que todas ellas son equivalentes e incluso que todas en definitiva carecen de sentido, pues, desde este punto de vista, no puede darse realmente ninguna explicación adecuada, y ninguna verdadera distinción puede hacerse entre las infinitas interpretaciones. La referencia a la hermenéutica aparece, pues, como innecesaria. El trabajo hermenéutico aquí realmente no lleva a ninguna comprensión o inteligibilidad mayor $(2014,43)$.

$\mathrm{Y}$, como bien destaca la comentarista, esto tiene un peso decisivo en algunos casos, como el derecho de las víctimas ante un acontecimiento histórico. Según Gschwandtner, la "certeza negativa" no puede ser absoluta en estos casos y la hermenéutica debe cumplir una función más decisiva que la de una mera "hermenéutica infinita". En palabras de Gschwandtner:

Aunque nuevas interpretaciones son siempre posibles y bien pueden ser esclarecedoras, no todas las interpretaciones son posibles y, ciertamente, no todas las interpretaciones son iguales. [...] La concepción marioniana de la hermenéutica parece una abdicación de la responsabilidad en vez de un llamado al discernimiento $(2014,47)$.

Sin embargo, la respuesta para esta objeción se encuentra en los diversos niveles de la hermenéutica marioniana. En primer lugar, en el primer nivel, el adonado acepta la responsabilidad mayor al discernir mediante un compromiso en primera persona -que no es solo teórico, sino eminentemente práctico- que allí hay una llamada dirigida a él. Esto implica que el adonado acepta que es responsable no solo por todos los otros adonados, sino por todo fenómeno que le sale al encuentro. Y, si bien puede objetarse qué sentido tiene hacerse responsable por "todo fenómeno" si no es porque éstos en definitiva reenvían a los adonados, entiendo que esta ampliación de la responsabilidad introduce una importante diferencia, pues implica escuchar una llamada y -yendo más allá de Lévinas- permite ver directamente un rostro también

10 Marion destaca en Certitudes négatives, que ante los fenómenos saturados es posible alcanzar un tipo de certeza que se caracteriza como "negativa". "Si una cuestión dotada de sentido, correctamente formulada y sin contradicción lógica, queda sin respuesta posible para un espíritu finito, e incluso no debe, por razones a priori, recibir respuesta según los criterios de una racionalidad finita (metafísica, los dos principios de contradicción y de razón suficiente), entonces esa cuestión en cuanto siempre es buscada y siempre dejada sin respuesta, que sobrevive sin embargo a esta ausencia ¿acaso no da ella una realidad que pensar (cogitable) y no merece el rango de certeza negativa?" (Marion, 2010b, 19-20). 
en el perro, en el árbol, y hasta en la montaña; implica un habitar el mundo ya no desde la actitud dominadora del sujeto, sino desde el profundo respeto del adonado. Entonces, en el primer nivel, el adonado, al aceptar la llamada, discierne que todo fenómeno es originariamente un fenómeno saturado. En este sentido, ya en este nivel inicial encontramos un uso hermenéutico del discernimiento que nos permite alcanzar una mayor comprensión e inteligibilidad. En palabras de Marion:

Esta decisión de tomar las cosas como llamadas -o la de no escuchar una voz cuando esto nos viene bien para justificar el permanecer en nosotros mismos- decide respecto de todo lo demás. La más alta lucidez consiste en identificar la llamada $(2012,70)$.

Pero, además, en el segundo nivel de hermenéutica, el nivel de la "hermenéutica de la variación" (identificado como el cuarto uso) se discierne -a partir de un compromiso con la situación-si en el caso concreto se está ante un objeto o ante un acontecimiento, es decir, si se debe constituir un objeto o entregarse al acontecer del fenómeno. En numerosos casos de la cotidianeidad, como reconoce explícitamente Marion, es necesaria la objetivación para no impedir "la vida social" ( $c f$. 1998, 437). Por ejemplo, un cirujano que tiene que realizar una operación debe objetivar el cuerpo de ese existente humano. Este tipo de hermenéutica permanece activa, pues puede ocurrir que un fenómeno interpretado como objeto, en cierto momento, requiera una "variación hermenéutica" que permita verlo como acontecimiento. Continuando con el ejemplo de la intervención quirúrgica, el paciente en recuperación, que experimenta los dolores posoperatorios, demanda ser fenomenalizado como rostro y ya no como objeto.

En el tercer nivel de hermenéutica, en el nivel de la "hermenéutica de la gradualidad" (tercer uso), Marion introduce explícitamente la posibilidad de un trabajo de discernimiento entre diversos grados y matices de objetidad y de saturación también. El adonado enfrenta, en este nivel, la tarea de discernir el grado de objetidad o de acontecialidad (saturación) que corresponde a ese caso concreto, a partir de la primera tópica de los fenómenos o introduciendo una nueva tópica con más distinciones si fuera necesario. Ciertamente, el tipo de discernimiento y criterio varía si se está en el campo de los objetos o en el campo de los acontecimientos. Para los objetos rigen diversas modalidades de la racionalidad del cálculo en un sentido amplio o, si se prefiere, la racionalidad del pascaliano orden del espíritu, ${ }^{11}$ mientras que para los acontecimientos en necesaria la racionalidad de lo incalculable, la racionalidad del orden del corazón, es decir, la lógica del amor.

Asimismo, como hemos analizado, Gschwandtner critica la falta de una efectiva práctica de la distinción de grados por parte de Marion (cf. 2014, 4-6), así como

11 Marion no menciona ningún criterio, pero si tenemos en cuenta que adhiere expresamente a la propuesta de relación entre fenomenología y hermenéutica presentada por Claude Romano en el capítulo XXII de $A u$ cœur de la raison, la phénoménologie, probablemente no estaría en desacuerdo con los criterios que el autor señala como propios de diversas hermenéuticas para distinguir entre mejores o peores interpretaciones: 1) la coherencia, 2) la potencia (la capacidad de dar cuentas de la mayor parte de los aspectos de la cosa en cuestión), 3) el enraizamiento histórico (que evita anacronismos), 4) la radicalidad de las preguntas formuladas, 5) la originalidad que se basa en el conocimiento de otras interpretaciones (Romano, 2010, 900). Ciertamente, la enumeración de Romano no es exhaustiva, pueden sumarse otros criterios que introduzcan motivos racionales para optar por una u otra interpretación. En particular, respecto de las víctimas de la historia, pueden considerarse criterios éticos de diverso tipo, aunque la última palabra en estos casos - para Marion- la tendría la lógica del amor que prescribiría ver un rostro en cada víctima. Volveré sobre esta cuestión en el cuerpo del texto. 
también critica la ausencia de una dimensión preparatoria en la hermenéutica marioniana ( $c f$. 2014, 90). Ambas críticas tienen su pertinencia, pues es cierto que Marion no se detiene demasiado en ninguna de estas dos cuestiones. No obstante, existen algunas mínimas indicaciones y lineamientos presentes en su obra. Marion sostiene que la fenomenicidad saturada no depende solamente de lo dado, sino también del modo que el adonado "la recibe, la experimenta y la expresa: en resumen, de que él sepa interpretarla” $(2016,95)$. Esta interpretación exige cierta capacidad por parte del intérprete:

La interpretación varía con el talento, la educación, y el coraje también, es decir, con la resistencia que el adonado pueda, cada vez, desplegar para recibir lo dado. Tal resistencia -que, en un sentido, consiste en no resistir, sino más bien en soportar la insistencia de lo dado-depende de la hermenéutica $(2014,95)$.

Contrariamente a lo objetado por Gschwandtner, puede entenderse que con el término "educación" Marion señala la necesidad de cierta preparación, y de cierto conocimiento de la historia y el contexto del fenómeno. Como bien señala Gschwandtner:

Si se proveen un entrenamiento, una preparación y un contexto adecuados, un fenómeno puede ser experimentado como saturado cuando no sería experimentado como tal sin esa preparación y contexto $(2014,91)$.

Si bien es cierto que se trata de un aspecto poco desarrollado y al que habría que añadir la complejidad de un "entrenamiento" y una "preparación" para ver lo que no puede ser previsto (el fenómeno saturado), considero que Marion, en buena medida, aceptaría la sugerencia de Gschwandtner. El adonado debe prepararse para asumir la responsabilidad por una interpretación que abre -en el primer nivel, pero que se refleja en todos los niveles- la posibilidad de toda otra interpretación, o de una interpretación realmente adecuada:

El fenómeno saturado exige una hermenéutica en la que el "en tanto que" existencial acepte exponerse a la contra-experiencia, por tanto, acepte llevar a cabo un combate contra la interpretación inevitablemente objetivante, dictada por el "en tanto que" apofántico (Marion, 2016, 95).

El adonado se enfrenta a este conflicto hermenéutico decisivo. No todas las interpretaciones son iguales, pero la diferencia fundamental está en una elección que se da en términos dicotómicos entre la clausura del objeto o la apertura a sus posibilidades. Sólo si el fenómeno es concebido desde esa apertura, es posible articular la necesidad de gradación y de jerarquización entre interpretaciones, e incluso, en ciertos casos, por "variación hermenéutica", aceptar la clausura en la objetidad. Pues, efectivamente, como ya hemos señalado, la modalidad del objeto es necesaria.

Conviene pues primero asegurar el primer nivel de hermenéutica, en el que asumiendo la responsabilidad que esto implica, se otorga a la llamada su carácter de tal. En ese primer nivel (que se refleja también en el segundo nivel, con la posibilidad de un pasaje dicotómico entre objeto y acontecimiento) se juega la decisión 
fundamental entre la clausura o la apertura, entre la mismidad o la otredad, entre la totalidad o el infinito.

En el tercer nivel se introducen grados, distinciones, jerarquías, teniendo en cuenta como criterio último la originariedad y la prioridad del carácter acontecial de todo fenómeno, pero también la necesidad, en ciertos casos, de una objetivación. Finalmente, en el cuarto nivel se constata la infinitud del campo abierto por la saturación y la posibilidad siempre abierta de otra interpretación.

En el caso concreto -señalado con buen tino por Gschwandtner- de un acontecimiento histórico en el que está en juego la reivindicación de las víctimas, puede ocurrir que sea necesario introducir una "variación hermenéutica", que transforme el acontecimiento en objeto, para considerar desde una lógica metafísica qué interpretación objetivante es la más adecuada para lograr esa reivindicación. Pero cabe señalar que esta operación estaría subordinada a una consideración de las víctimas como rostros, como fenómenos saturados, como acontecimientos, alcanzada por la decisión hermenéutica fundamental por la que el adonado se entrega a otra racionalidad: a la lógica del amor.

\section{Referencias bibliográficas}

Benoist, J. (2016) Logique du phénomène. Paris: Hermann.

Gadamer, H.-G. (1999) Gesammelte Werke. Band 1: Hermeneutik I. Wahrheit und Methode. Tübingen: Mohr Siebeck.

Greisch, J. (1991) “L’herméneutique dans la 'phénoménologie comme telle': trois questions à propos de 'Réduction et donation"”. Revue de métaphysique et de morale 96: 43-63.

Greisch, J. (1999) "Index sui et non dati: Les paradoxes d'une phénoménologie de la donation". Transversalités: revue de l'Institut Catholique de Paris 70: 27-54.

Grondin, J. (1992) "La phénoménologie sans herméneutique: Jean-Luc Marion, Réduction et donation". Internationale Zeitschrift für Philosophie 1: 146-53.

Grondin, J. (1999) "La tension de la donation ultime et de la pensée herméneutique de l'application chez Jean-Luc Marion”. Dialogue. Revue canadienne de philosophie, 38: 547-559.

Gschwandtner, C. (2014) Degrees of Givenness. On saturation in Jean-Luc Marion. New York: Fordham University Press.

Habermas, J. et al. (1971) Hermeneutik und Ideologiekritik. Frankfurt am Main: Suhrkamp.

Heidegger, M. (1977) Gesamtausgabe. I. Abteilung: Veröffentlichte Schriften 1914-1970. Band 2. Sein und Zeit. Frankfurt am Main: Vittorio Klostermann (GA 2).

Henry, M. (1991), “Quatre principes de la phénoménologie". Revue de Métaphysique et de Morale. À propos de Réduction et donation de Jean-Luc Marion, 96, 1: 3-26.

Husserl, E. (2006) Husserliana Materialien VIII. Späte Texte über Zeitkonstitution (19291934): Die C-Manuskripte. Dordrecht: Springer (HuaM VIII).

Marion, J.-L. (1989) Réduction et donation. Recherches sur Husserl, Heidegger et la phénoménologie. Paris: PUF.

Marion, J.-L. (1998) Étant donné. Essai d'une phénoménologie de la donation, seconde édition corrigé. Paris: PUF.

Marion, J.-L. (2005a) « Entretien avec Jean-Luc Marion. Vincent Citot et Pierre Godot », Le Philosophoire, numéro 11 « L'amour »: 5-22.

Marion, J.-L. (2005b) Le visible et le révélé. Paris: Cerf. 
Marion, J.-L. (2010a) De surcroît. Études sur les phénomènes saturés, première édition « Quadridge ». Paris: PUF.

Marion, J.-L. (2010b) Certitudes négatives. Paris: Grasset.

Marion, J.-L. (2012) La rigueur des choses. Entretiens avec Dan Arbib. Paris: Flammarion.

Marion, J.-L. (2016) Reprise du donné. Paris: PUF.

Nietzsche, F. (1967) Unzeitgemässe Betrachtungen II en Nietzsche, F., Kritische Studienausgabe I, herausgegeben von Giorgio Colli und Mazzino Montinari. Berlin/New York: Walter de Gruyter (KSA I).

Ricœur, P. (1986) Du texte à l'action. Essais d'herméneutique II. Paris: Seuil.

Ricœur, P. (2013) Le conflit des interprétations. Essais d'herméneutique. Paris: Seuil.

Romano, C. (2010) Au cour de la raison, la phénoménologie. Paris: Gallimard.

Serban, C. (2012) "La méthode phénoménologique, entre réduction et herméneutique". Les études philosophiques 100, 1: 81-100.

Walton, R. J. (2015) Intencionalidad y horizonticidad. Bogotá: Aula de Humanidades.

Welton, D. (1983) The Origins of Meaning. A Critical Study of the Thresholds of Husserlian Phenomenology. The Hague: Martinus Nijhoff. 